\title{
Encefalomielitis aguda diseminada y colitis ulcerosa: descripción de un caso clínico
}

\section{Acute disseminated encephalomyelitis and ulcerative colitis: description of a clinical case in magnetic resonance}

\author{
Jorge Guzmán S. ${ }^{1}$ y Rodrigo Salinas R. ${ }^{2}$
}

\begin{abstract}
Acute disseminated encephalomyelitis (ADEM) is an acute demyelinating disorder of the central nervous system, characterized by an acute or subacute onset of neurological symptoms and focal signs in association with multifocal demyelinating findings on neuroimaging. Spontaneous resolution has been reported within days of onset, corticosteroids therapy accelerates the resolution time. ADEM has been associated with previous infections, and its relationship with other autoinmune disease is unclear. A case of ADEM is reported, in a patient previously diagnosed with ulcerative pancolitis, whose neurological symptoms resolved after 3 days of hospital admission without evident sequelae after receiving a short empirical treatment with ampicilin, ceftriaxone, acyclovir and intravenous steroids.
\end{abstract}

Key words: Acute disseminated encephalomyelitis, ulcerative colitis, case report.

Rev Chil Neuro-Psiquiat 2012; 50 (1): 57-61

\section{Introducción}

$\mathrm{L}^{\mathrm{a}}$ a encefalomielitis aguda diseminada (EAD) es un trastorno desmielinizante agudo que afecta al sistema nervioso central. Se caracteriza por la aparición aguda o subaguda de síntomas y signos neurológicos, característicamente encefalopatía, signos focales o multifocales como hemiparesia, paraparesia, compromiso de pares craneales, meningismo, ataxia u otros trastornos del movimiento ${ }^{1}$. Existen hallazgos multifocales de desmielinización en las neuroimágenes, que son variables, hiperintensas en T2 y FLAIR y típicamente ubicadas en la sustancia blanca subcortical, aunque el compromiso de sustancia gris, especialmente tálamo y ganglios basales, no es infrecuente ${ }^{1,2}$. Ocurre mayormente en la infancia y adolescencia, aunque en la literatura se describe reportes de casos en adultos. La patogenia de la EAD es incierta, sugiriéndose una reacción autoinmune a un agente infeccioso, en la forma de mimetismo molecular contra la proteína básica de la mielina u otro autoantígeno ${ }^{2,3}$, toda vez que alrededor de un $50-75 \%$ de los casos se presenta luego de una infección viral o bacteriana, frecuentemente del tracto respiratorio superior $^{4,5}$. Esta característica, sin embargo, no se encuentra en los criterios diagnósticos aceptados, dada por la proporción significativa de casos que carecen de este antecedente ${ }^{1}$. Se ha descrito casos de EAD en el contexto de enfermedades autoinmu-

Recibido: 5/09/2011

Aprobado: 12/03/2012

1 Interno de Medicina, Facultad de Medicina Universidad de Chile, Santiago, Chile.

2 Servicio de Neurología, Hospital del Salvador. Santiago, Chile. 
nes, como lupus, anemia hemolítica autoinmune y esclerosis múltiple, aunque las publicaciones en este sentido son escasas. Se presenta un caso de $\mathrm{EAD}$ en un paciente masculino con diagnóstico previo de pancolitis ulcerosa.

\section{Caso clínico}

Paciente de 21 años, con antecedentes de pancolitis ulcerosa diagnosticada en agosto de 2010, en tratamiento esteroidal con prednisona $60 \mathrm{mg} /$ día y Mesalazina 1 gr c/6 hrs durante 5 meses previos al ingreso. Días previos al diagnóstico completó tratamiento con ciprofloxacino por síndrome diarreico presuntamente bacteriano, sin confirmación etiológica y con respuesta favorable. Ingresó al Servicio de Urgencia del Hospital Del Salvador por cuadro de 48 horas de evolución de compromiso de conciencia caracterizado por desorientación temporo-espacial y somnolencia, además de deposiciones sanguinolientas. $\mathrm{Al}$ examen físico general presentaba parámetros hemodinámicos dentro de rangos normales, afebril y como único hallazgo se describe candidiasis orofaríngea. Al examen neurológico el paciente se encuentra vigil, con tendencia a la somnolencia, desorientado en tiempo y espacio, nistagmo vertical, prueba de pequeña paresia positiva a derecha, y signo de Brudzinski. Resto del examen sin hallazgos. Se realiza TAC de cerebro que no muestra hallazgos patológicos y se realiza punción lumbar que da salida a líquido cefalorraquídeo claro, con proteínas $74,8 / \mathrm{mm}^{3}$, glucorraquia normal, glóbulos rojos $2 / \mathrm{mm}^{3}$, leucocitos $760 /$ $\mathrm{mm}^{3}$ de predominio linfocítico. Por antecedente de inmunosupresión crónica y candidiasis orofaríngea al ingreso, se inicia tratamiento antibiótico con ampicilina+ceftriaxona+aciclovir y corticoides en dosis de estrés (Hidrocortisona 100mg c/8 hrs), y se traslada a Unidad de Intermedio. Evoluciona en buenas condiciones generales, estable desde el punto de vista hemodinámico y recuperando conciencia a las 48 horas. Se realiza Resonancia magnética (Figura 1) que muestra imágenes con compromiso talámico bilateral asimético asociado a compromiso capsular posterior izquierdo con discreta captación de contraste.

Posteriormente se traslada a sala común, evolucionando de manera estable y con algunos episodios de hemorragia digestiva baja escasa. Se completa estudio infeccioso por cuadro gastrointestinal, con leucocitos fecales, coprocultivo, parasitológico seriado, toxina para $C$. difficile y antigenemia para Citomegalovirus, todos ellos dentro de límites normales. Es evaluado por equipo de Gastroenterología, quienes diagnostican cuadro de colitis ulcerosa activa por lo que se agrega azatioprina a la terapia.

Se controla en una semana y un mes posterior al egreso, permaneciendo asintomático desde el punto de vista neurológico y con examen físico dentro de rangos normales.
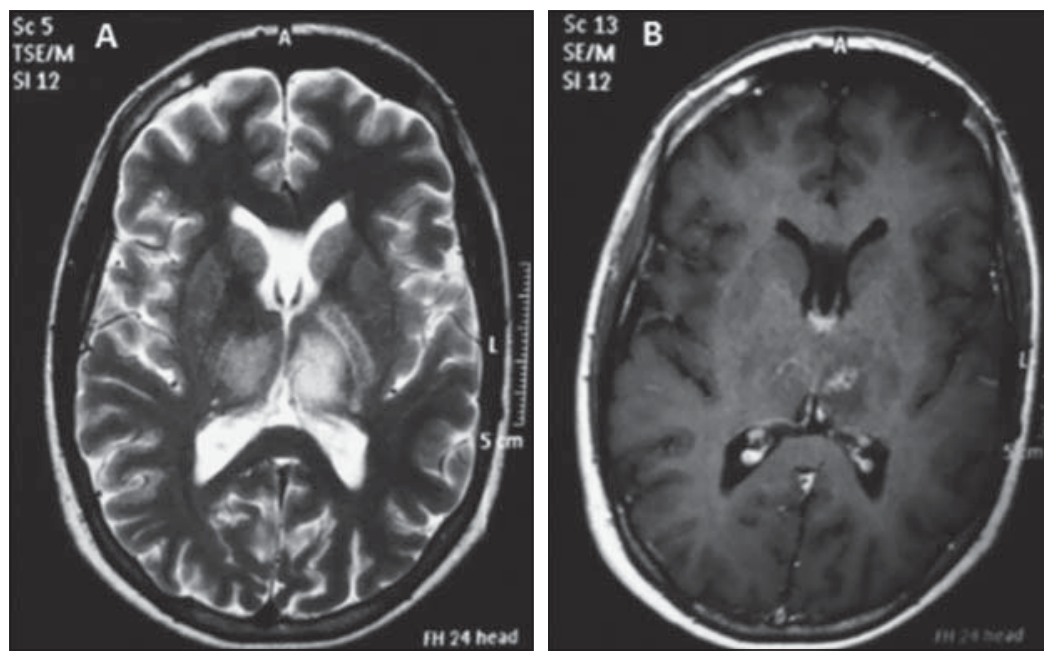

Figura 1. Resonancia magnética de cerebro. A) Imagen ponderada en T2 con hiperintensidad en ambos tálamos, mayor a izquierda y compromiso capsular posterior izquierdo. B) Imagen ponderada en $\mathrm{T} 1$ con contraste que muestra discreta captación de gadolinio en tálamo izquierdo. 


\section{Discusión}

La colitis ulcerosa (CU) es una enfermedad crónica caracterizada por inflamación de la mucosa colónica. En consideración con su patogenia, además de una predisposición genética y una excesiva respuesta a antígenos dietarios frente a un agente infeccioso no identificado, actualmente se plantea la posibilidad de una alteración en la regulación de la respuesta inmune ${ }^{6}$. Además de la afectación colónica, se describe que en al menos el 50\% de los pacientes, durante una vez en su vida, desarrollarán una o más manifestaciones extraintestinales. En este sentido, se describen en la literatura manifestaciones del sistema nervioso central como tromboembolismo y enfermedad cerebrovascular, epilepsia, mielopatía, vasculitis cerebral y enfermedades desmielinizantes ${ }^{7}$. En relación a estas últimas, se ha asociado la CU a esclerosis múltiple tanto en estudios epidemiológicos como desde un punto de vista de hallazgos en las neuroimágenes ${ }^{8}$. Sin embargo, como los criterios actuales de EM son más exactos que los anteriormente aplicados en esos estudios, es probable que exista una relación entre enfermedades desmielinizantes tipo EM, como la EAD, y colitis ulcerosa ${ }^{7}$.

En el caso expuesto, el paciente tiene antecedentes de un cuadro digestivo bajo previo, cuyo estudio resulta negativo para demostrar infección al igual que el cuadro actual, tras documentar cultivos de deposiciones, parasitológico seriado y estudios de antigenemia para citomegalovirus. En el contexto de EAD, resulta relevante hacer notar la negatividad de los estudios para infección intestinal, pues hay reportes de casos que han relacionado episodios de EAD a infecciones por Citomegalovirus ${ }^{9,10}$ y Campylobacter jejuni ${ }^{11,12}$. En la anamnesis, el paciente no refiere cuadro respiratorio alto previo, lo que se ha correlacionado con estudios que describen este antecedente en casos de EAD particularmente en la población pediátri$\mathrm{ca}$, siendo mucho más infrecuente en adultos ${ }^{13}$. El paciente inició su sintomatología neurológica en forma aguda, sin que se documentara infección del sistema nervioso central, encontrándose como hallazgo la presencia de pleocitosis de predominio linfocítico, que ha sido descrito en la literatura en casos de $\mathrm{EAD}^{1,5}$, y con resonancia magnética que muestra hallazgos compatibles con EAD. En la literatura se describen como características sugerentes de EAD, la presencia de lesiones multifocales, hiperintensas y frecuentemente bilaterales de forma asimétrica, con imágenes en T2 y FLAIR, mayores a $1 \mathrm{~cm}$ que comprometen sustancia blanca supra e infratentorial, y/o compromiso de tálamo y ganglio basales ${ }^{2}$.

En cuanto a la evolución del cuadro neurológico, el paciente se encuentra asintomático a los 3 días del ingreso. Durante su evolución recibe tratamiento antibiótico y corticoides endovenosos en dosis de estrés por su inmunosupresión crónica. Actualmente sólo se dispone de evidencia empírica que avala el tratamiento con metilprednisolona endovenosa como de primera línea, al igual que el uso de inmunoglbulina endovenosa, tradicionalmente indicada en aquellos casos con alta sospecha de meningo-encefalitis o fracaso de tratamiento con metilprednisolona ${ }^{14}$. No hay evidencia publicada que muestre claramente el impacto y la superioridad de uno respecto a otro en pacientes con EAD, pero se cree que ambos acelerarían la fase de recuperación inicial, y en cuanto a las implicancias en el pronóstico a largo plazo, la evidencia es mucho menor. También se han reportado casos de mejoría clínica con el uso de plasmaféresis en pacientes pediátricos que no responden al uso de corticoides ni inmunoglobulina endovenosos, bajo la premisa de remover los anticuerpos responsables del proceso autoinmunitario; sin embargo, la serie más larga es retrospectiva y reporta beneficio clínico en 6 de 13 pacientes pediátricos con diagnóstico clínico de EAD con mala respuesta a metilprednisolona endovenosa y como su uso es consideradado de rescate, no ha sido probado en pacientes que presentan una corta evolución de su enfermedad ${ }^{2,15}$. Además, es pertinente comentar que se ha descrito casos con resolución espontánea de los síntomas a los pocos días luego de la presentación aguda. También, es destacable que el paciente desarrolla el compromiso neurológico estando en tratamiento corticoesteroidal, en conocimiento que sería terapéutico y protector en los casos de $\mathrm{EAD}^{7}$; sin 
embargo, se desconoce su relación con el grado de actividad de la CU.

Por último, es interesante destacar la relación temporal entre la agudización de la CU y el episodio desmielinizante agudo, lo que podría relacionarse con mecanismos patológicos similares por cuanto la CU se caracteriza por una alteración en la función de barrera epitelial que favorece la captación de antígenos luminales y la estimulación de una reacción inmune e inflamatoria patológicas ${ }^{16}$, llegándose a plantear incluso como una varian- te crónica de un estado pre-desmielinizante, en extrapolación a lo que sucede con los gatillantes infecciosos en la $\mathrm{EAD}^{7}$. Hay evidencia que muestra que las manifestaciones neurológicas en pacientes con CU no se correlacionan con el grado de actividad de la enfermedad ${ }^{17}$, sin embargo, estos estudios engloban la totalidad de manifestaciones neurológicas en estos pacientes y no a la EAD como entidad particular, de modo que para establecer esta relación con mayor certeza se requieren de estudios adicionales.

\begin{abstract}
Resumen
La encefalomielitis aguda diseminada (EAD) es un trastorno desmielinizante agudo del Sistema Nervioso Central, caracterizado por la presentación aguda de sintomas y signos neurológicos en asociación con hallazgos multifocales de desmielinización en las neuroimágenes. Se describe en la literatura resolución espontánea a los pocos días de presentación, con secuelas variables. La terapia corticoesteroidal aceleraría este tiempo de resolución. Se ha relacionado con eventos infecciosos previos, y su relación con otras enfermedades autoinmunes no es clara. Se presenta un caso de EAD en paciente con diagnóstico previo de pancolitis ulcerosa cuya sintomatología neurológica se resuelve tras 3 días del ingreso hospitalario, sin secuelas evidentes, tras recibir tratamiento empírico corto con Ampicilina, Ceftriaxona, Aciclovir y corticoides endovenosos en dosis de estrés.
\end{abstract}

Palabras clave: Encefalomielitis Aguda Diseminada, colitis ulcerosa, caso clínico.

\section{Referencias}

1. Young NP, Weinshenker BG, Lucchinetti CF. Acute Disseminated Encephalomyelitis: Current Undertstanding and Controversies. Semin Neurol 2008; 28 (1): 84-94.

2. Noorbakhsh F, Johnson R, Emery D, et al. Acute Disseminated Encephalomyelitis: Clinical and Pathogenesis Features. Neurol Clin 2008; 26: 759-80.

3. Garg RK. Acute disseminated encephalomyelitis. Postgrad Med J 2003; 79 (927): 11-7.

4. Tenembaum S, Chamoles N, Fejerman N. Acute disseminated encephalomyelitis: a long-term follow-up study of 84 pediatric patients. Neurology 2002; 59: 1224.

5. Schwarz S, Mohr A, Knauth M, Wildemann B,
Storch-Hagenloche B. Acute disseminated encephalomyelitis: a follow-up study of 40 adult patients. Neurology 2001; 56 (10): 1313-8.

6. Sartor RB. Mechanisms of disease: Pthogenesis of Crohn's disease and ulcerative colitis. Nat Clin Pract Gastroenterol Hepatol 2006; 3 (7): 390-40.

7. Scheid R, Teich N. Neurologic manifestations of ulcerative colitis. Eur J Neurol; 14 (5): 483-93.

8. Hart PE, Gould SR, MacSweeney JE, et al. Brain white-matter lesions in inflammatory bowel disease. Lancet 1998; 335: 1158.

9. Revel-Vilk S, Hurvitz H, Klar A, Virozov Y. Recurrent acute disseminated encephalomyelitis associated with acute cytomegalovirus and EpsteinBarr virus infection. J Child Neurol 2000; 15 (6): 421. 
10. Zaguri R, Shelef I, Ifergan G, et al. Fatal acute disseminated encephalomyelitis associated with cytomegalovirus infection. BMJ Case Reports 2009, Mar.

11. Orr D, McKendrick M, Sharrack B. Acute disseminated encephalomyelitis temporally associated with Campylobacter gastroenteritis. J Neurol Neurosurg Psychiatry 2004; 75 (5): 792-3.

12. Young JW, Mason DF, Taylor BV. Acute inflammatory encephalomyelitis following Campylobacter enteritis associated with high titre antiganglioside GM1 IgG antibodies. J Clin Neurosci 2009; 16 (4): 597-8.

13. Wang PN, Fuh JL, Liu HC, Wang SJ. Acute disseminated encephalomyelitis in middle-aged or elderly patients. Eur Neurol 1996, 36: 219-23.
14.- Sahlas DJ, Miller SP, Guerin M, et al. Treatment of Acute Disseminated Encepgalomyelitis with intravenous immunoglobulin. Neurology 2000; 54: 1370.

15. Khurana DS, Melvin JJ, Kothare SV, et al. Acute disseminated encephalomyelitis in children: discordant neurologic and neuroimaging abnormalities and response to plasmapheresis. Pediatrics 2005; 116: 431.

16. Velin AK, Ericson AC, Braaf Y, et al. Increased antigen and bacterial uptake in follicle associated epithelium induced by chronic stress in rats. Gut 2004; 53: 494-500.

17. Lossos A, Alieakim A, Steiner I. Neurologic aspects of inflammatory bowel disease. Neurology 1995; 45: 416-21.
Correspondencia:

Jorge Guzmán Sanders

Dragones de la Reina 773, casa G

La Reina, Santiago, Chile.

Fonos: 2277297 - 90746071

E-mail: jguzmans.med@gmail.com 